\title{
Phase Characterization of Two-Phase BiSn Alloy Nanoparticles Using Conventional TEM and EFTEM Spectral Imaging Techniques
}

\author{
C.T. Schamp, ${ }^{*}$ W-S Cheng, ${ }^{* *}$ and W.A. Jesser** \\ *CeriumLabs, 5204 E. Ben White Blvd, M/S 512, Austin, TX, 78741, USA \\ **Department of Materials Science and Engineering, University of Virginia, 395 McCormick Rd., \\ Charlottesville, VA 22904, USA
}

The size dependence of material properties offers an additional degree of freedom for engineering applications of the future. Many material properties are size dependent including the melting temperature, lattice parameters, and solubility limits [1].Here, we report on alloy nanoparticles of Bi and Sn grown through thermal co-evaporation/deposition and analyzed through analytical (scanning) transmission electron microscopy ((S)TEM) methods.

A home built ultra-high vacuum deposition system is used to simultaneously evaporate $\mathrm{Bi}$ and $\mathrm{Sn}$ from tungsten wire baskets onto heated TEM grids supporting an approximately $2 \mathrm{~nm}$ thick continuous amorphous carbon film (Ted Pella \#01824). The grids were held at about $90^{\circ} \mathrm{C}$ during and for about an hour after the deposition to enhance atomic mobility and equilibrium nucleation and growth of alloy nanoparticles [2]. The grids were allowed to cool, and were withdrawn from the deposition system to be placed in plastic membrane boxes and shipped for analytical TEM analysis.

The (S)TEM analysis utilized a Philips/FEI CM300 (S)TEM operated at 300kV, equipped with a model 2002 Gatan Imaging Filter (GIF), and a Noran X-ray detector. Gatan's Digital Micrograph software is used to digitally capture and process conventional TEM and Energy Filtered TEM (EFTEM) data. The Noran System Six software package is used to acquire and process STEM images, and STEM-based energy dispersive X-ray spectroscopy (EDXS) data.

A typical bright field TEM micrograph of an alloy distribution is shown in figure 1, with a selected area diffraction pattern inset encompassing the same area as the image. Diffraction contrast suggests more than one phase or grain is possible within a particle, however the diffraction pattern shows only a single set of rings which is consistent with the crystal structure of bulk Bi. Figure 2 shows a high resolution TEM micrograph of a particle from the same sample as figure 1 showing two grains in the same particle surrounded by an amorphous layer. Two Hanning window filtered FFT's were extracted from figure 2 at the locations of the boxes. The Fourier component intensities were false colored in red for the upper box and blue for the lower box and superimposed on the FFT of the upper box. The red box is consistent with a [-22-1] zone axis of $\mathrm{Bi}$, and the blue box is consistent with the $\{012\}$ periodicity of $\mathrm{Bi}$. Assuming that two particles were coalescing, it is interesting that they did not form a single crystal, possibly due to a smaller particle within the interface.

Figure 3 shows a composite image from a principle component analysis of STEM-EDXS data, with the component spectra given below indicating a $\mathrm{Sn}$ rich oxide phase segregating to the surface with a Bi-rich core. An MLLS fit to a low-loss EFTEM spectral image using the reference spectra shown confirms the existence of two phases that are consistent with a core-shell type model as seen in figure 4. The data supports the notion that the nanoparticles have a crystalline Bi-rich core surrounded by an amorphous Sn-rich oxide shell. 
[1] G.B. Sergeev, Nanochemistry, Elsevier Science, Amsterdam, 2006

[2] C.T. Schamp and W.A. Jesser, Met Trans A, 37A (2006) 1825
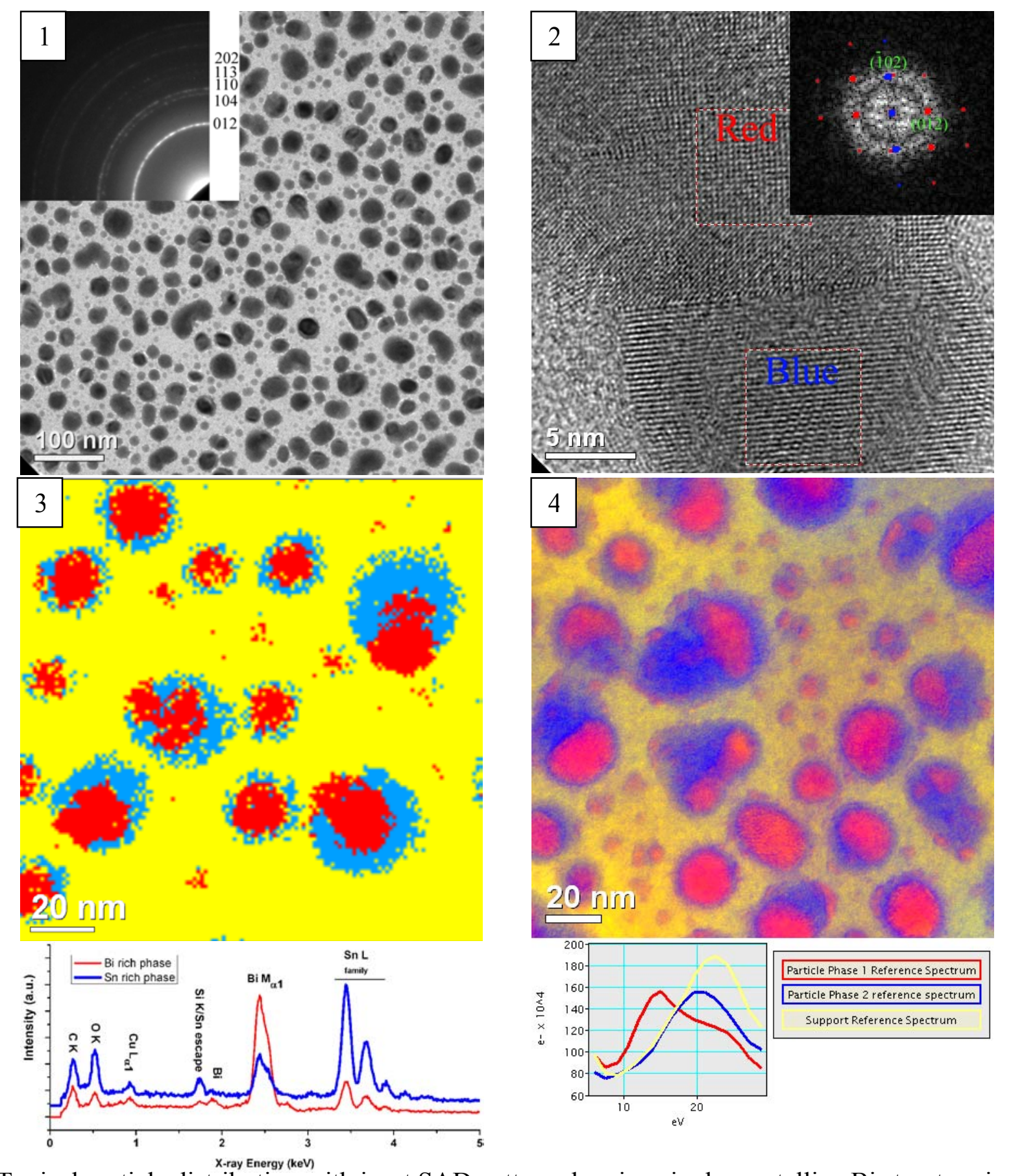

Typical particle distribution with inset SAD pattern showing single crystalline Bi structure in figure 1. HRES image of two-grain poly crystal with indexed [2-21] Bi Weiner filtered FFT inset. STEM-EDXS principle component phase map illustrating Sn-rich oxide segregating to the surface surrounding a Bi core in figure 3 and low loss MLLS fit EFTEM phase map confirming phase distribution within and around particles in figure 4. 